ocean-atmosphere climatic models is apparent from the discussion by Jill Williams on modelling general atmospheric circulation. Results of four models of the last ice age maximum are compared. Robert Chervin discusses the limitations of general circulation models. He shows how statistical methods are used to separate the signal and noise components of a model's output. An intuitive judgement has to be made in many cases.

The final section of the book discusses how general circulation models can be used to study the interaction of man with today's climate, and what climatic changes this might produce in the future. Will Kellogg examines the effects of increasing land cultivation, fossil fuel consumption and other industrial contaminants. He concludes that climate will generally become warmer and wetter, especially over the land, and this trend may already be overtaking natural climatic trends. Kellogg suspects that this warming might greatly reduce or even eliminate the arctic pack ice, but sees no reason to think it could affect the stability of present ice sheets until far into the future. Stephen Schneider and Richard Temkin discuss climatic changes and human affairs, particularly food, water, and energy resources.

Terence J. Hughes

Terence J. Hughes is an Associate Professor in the Department of Geological Sciences at the University of Maine, Orono.

\section{Broad survey of diffraction}

Diffraction From Materials. By L. H. Schwartz and H. B. Cohen. Pp. 558. (Academic: New York, San Francisco and London, 1977.) \$27.50; £19.95.

Diffraction, especially X-ray diffraction, provides an experimental approach to a wide range of structural problems in such disparate disciplines as chemistry, biology and materials science. The importance of certain aspects of the subject has resulted in the growth of specialisations, and in chemistry, for example, the elucidation of molecular constitution, stereochemistry and geometry by X-ray studies of crystal structure is now a routine procedure with a large body of practitioners and an output of results that requires the Chemical Society Specialist Periodical Report Molecular Structure by Diffraction Methods to review more than 2,000 references annually.

There is a risk that scientists immersed in one aspect of diffraction may lose sight of the possibilities that exist for making use of the other aspects of the subject. Accordingly, in addition to the books devoted to the specialised areas of diffraction, there is a place for textbooks that provide a broad survey of the subject. Schwartz and Cohen succeed admirably in this latter aim.

Their book opens with an account of crystal symmetry and then deals with geometrical aspects of crystals and lattices Stereographic projections are described in some detail in the section on analytical calculations and graphical techniques. Chapter 3 is concerned with kinematical scattering theory, and discussion of diffraction from gratings, rows of atoms and threedimensional arrays leads to the derivation of the reciprocal lattice and of the structure factor Chapters 4 and 5 describe how beams of X-radiation, neutrons and electrons are obtained for diffraction experiments, how these beams interact with matter and how the scattered radiation is detected. The topics covered include absorption and dispersion effects, magnetic scattering of polarised neutrons, and singlecrystal and powder diffraction techniques.

The second half of the book develops three major topics. The determination of crystal structures is the theme of chapter 6, in which the authors introduce the principles of Fourier transforms. Electron-density mapping, the Patterson function, intensity statistics, direct phasing procedures and least-squares refinement are clearly explained. Chapter 7 is entitled "What Else Can We Learn from a Diffraction Experiment besides the Average Structure?" and devotes 71 pages to a discussion of thermal diffuse scattering, mosaic size, stacking disorder, local order and clustering, small-angle scattering, and diffraction by liquids and amorphous solids. The final chapter gives an exposition of the dynamical theory of diffraction and concludes with an outline of X-ray topography.

The book is well illustrated and there are useful references to other texts and to original papers. An excellent set of problems follows each chapter, providing the reader with a rigorous test of his mastery of the contents.

Schwartz and Cohen have provided us with one of the best general treatments of the subject currently available and $I$ can recommend their book to all scientists with an interest in diffraction experiments.

George Sim

George Sim is Gardiner Professor of Chemistry at the University of Glasgow, UK.

\section{Valence and molecular orbital theories}

Structures and Approximations for Electrons in Molecules. By D. B. Cook. Pp. 294. (Wiley: London, New York and San Francisco, 1978.) $£ 13.50$.

THE armoury of books available on valence and molecular orbital theories is so well stocked that some ingenuity is necessary for a new book in this area to be anything other than a rehash of earlier texts. The mere fact that Dr Cook has succeeded is a credit to his originality, and he convinces the reader that he is producing something quite novel. His earlier book on $a b$ initio valence calculations raises expectations which are not disappointed, and no-one could read the book without learning a great deal. The bcok is particularly important for the many who use semi-empirical molecular orbital methods.

Users of approximate methods are split into two camps: those whose main interest is molecular energetics and computed molecular properties, and those whose chief concern is the interpretation of valence theories. This book follows the second path. Much is made of the theoretical basis of the functional group approach, and the important but ambiguous role of molecular orbitals is clarified in a way not to be found in most books at this level (senior undergraduate and postgraduate students).

A particular strength is the emphasis on computational aspects. This matters, when so many workers use 'off the peg' programmes and only have a vague idea of the inner workings. Encouragement to play with the internal organs of programmes is provided by an Appendix entitled "Invent your own molecular orbital method"; a thought which might not please editors of journals but which should lead to understanding by the chemist.

The book is opinionated and expresses views which will not be universally accepted; but this only adds spice. It is not a polished product and is certainly easier to work from than to read, a conclusion based both on the content and on the production. Justified type is used in the preface and contents but thereafter we have unjustified reproduction of typescript.

Even at a cost above the photocopying limit the book deserves to be bought and used by individuals rather than referred to in libraries.

Graham Richards

Graham Richards is Lecturer in the Physical Chemistry Laboratory at the University of Oxford, UK. 